\title{
Characteristics of magnetospherically reflected chorus waves observed by CLUSTER
}

\author{
M. Parrot ${ }^{1}$, O. Santolík ${ }^{2}$, D. A. Gurnett ${ }^{3}$, J. S. Pickett ${ }^{3}$, and N. Cornilleau-Wehrlin ${ }^{4}$ \\ ${ }^{1}$ LPCE/CNRS, 3A Avenue de la Recherche, Orléans, 45071, France \\ ${ }^{2}$ Charles University, Faculty of Mathematics and Physics, V Holesovickach 2, Praha 8, 18000, Czech Republic \\ ${ }^{3}$ Department of Physics and Astronomy, University of Iowa, Iowa City, IA 52242-1479, USA \\ ${ }^{4}$ CETP/UVSQ, 10/12 Avenue de l'Europe, Velizy, 78140, France
}

Received: 4 September 2003 - Revised: 12 January 2004 - Accepted: 14 January 2004 - Published: 14 July 2004

Part of Special Issue "Spatio-temporal analysis and multipoint measurements in space"

\begin{abstract}
Chorus emissions are often observed by the STAFF spectrum analyser on board the 4 satellites of CLUSTER. This instrument provides the cross spectral matrix of three magnetic and two electric field components. Dedicated software processes this spectral matrix in order to determine the propagation characteristic of these chorus waves. Measurements of the parallel component of the Poynting vector around the magnetic equator indicate that the chorus waves propagate away from this region which is considered as the source area of these emissions. This is valid for the most intense waves observed on the magnetic and electric power spectrograms. But it has also been observed that lower intensity waves propagate toward the equator at the same frequency. Using the wave normal directions of these waves, a ray tracing study has shown that the waves have suffered a Lower Hybrid Resonance (LHR) reflection at low altitudes and now return to the equator at a different location with a lower intensity. The paper presents other similar events when WBD data are simultaneously recorded. The WBD experiment provides a much better time resolution and allows one to check the structure of the returning waves. It is observed that these waves have still a high degree of polarization, even if they started to lose the coherent structure of the chorus elements. They reach the equator with a small wave normal angle which is more efficient for a further amplification. It is explained that these emissions could be a source of hiss.
\end{abstract}

Key words. Magnetospheric physics (plasma waves and instabilities) - Ionosphere (wave propagation) - Radio science (magnetospheric physics)

\section{Introduction}

Chorus waves are intense whistler-mode emissions consisting of a sequence of discrete elements. The elements have a characteristic duration of a fraction of a second and they appear as rising and/or falling tones on power spectrograms. This type of emission has been extensively studied in the past (see the review paper by Sazhin and Hayakawa, 1992, and the references therein). Chorus is most probably generated by a nonlinear mechanism involving wave-particle interaction with energetic electrons (Kennel and Petschek, 1966; Helliwell, 1967; Tsurutani and Smith, 1974; Tsurutani et al., 1979; Meredith et al., 2001; Titova et al., 2003). Close to the source region, chorus is often divided into two frequency bands, above and below one-half of the electron cyclotron frequency (Tsurutani and Smith, 1974). Complex fine structure of chorus wave packets was recently found in their source region (Santolík et al., 2003b). The sources of chorus are located within a few thousands of $\mathrm{km}$ of the equatorial plane (Dunkel and Helliwell, 1969; Burtis and Helliwell, 1969; Burton and Holzer, 1974; Tsurutani and Smith, 1977; Nagano et al., 1996; LeDocq et al., 1998; Parrot et al., 2003a; Santolík et al., 2003b), with scales of the order of a few wavelengths perpendicular to the field lines (Santolík and Gurnett, 2003). Chorus thus propagates from its well localized source in the vicinity of the equatorial plane, but no evidence of reflected chorus waves returning to the equator was found until recently. The absence of reflected chorus was explained by strong Landau damping encountered by the chorus emissions propagating to high latitudes (Burton and Holzer, 1974; Goldstein and Tsurutani, 1984). 
Parrot et al. (2003b) have recently presented an event recorded on 29 October 2001, where magnetospherically reflected chorus waves are observed using ray tracing with the four CLUSTER satellites. With the wave normal directions of these waves, the ray tracing study shows that the waves observed by $\mathrm{SC} 1, \mathrm{SC} 2$ and $\mathrm{SC} 4$ cross the equatorial plane at the same location as the waves observed by SC3, which is $30 \mathrm{~min}$ late and first observes the waves which originate from the equator; meanwhile, SC1, SC2 and SC4 observe the same waves which have suffered a Lower Hybrid Resonance (LHR) reflection at low altitudes (based on the ray tracing analysis) and now return to the equator at a different location with a lower intensity. The event was observed with the SA (Spectrum Analyser) part of the STAFF (Spatio-Temporal Analysis of Field Fluctuations) experiment (Cornilleau-Wehrlin et al., 1997, 2000, 2003). STAFF-SA computes the spectral matrix of five components (three magnetic from STAFF plus two electric components from the Electric Field and Wave experiment EFW (Gustafsson et al., 2001) of the electromagnetic field up to $4 \mathrm{kHz}$. The SA produces a $5 \times 5$ spectral matrix with 27 frequencies distributed logarithmically between $8 \mathrm{~Hz}$ and $4 \mathrm{kHz}$. This frequency range is divided into three frequency sub-bands: A $(8-64 \mathrm{~Hz}), \mathrm{B}(64-512 \mathrm{~Hz})$, and C $(512-4000 \mathrm{~Hz})$ with different time intervals of analysis (for example, for one second of data, 64 spectra are averaged in the band C, 8 in the band B, and only one spectrum is considered in band A). After averaging, the time resolution of the spectral matrix varies between $125 \mathrm{~ms}$ and $4 \mathrm{~s}$. Therefore, STAFF-SA does not have enough time and frequency resolutions to verify the nature of the reflected waves, for example, to search for possible signs of discrete chorus elements. Another wave experiment WBD (Wideband) on board CLUSTER is therefore used. Among other possibilities, WBD provides continuous waveforms of one electric field component from $25 \mathrm{~Hz}$ up to $9.5 \mathrm{kHz}$ (Gurnett et al., 2001) during reduced time intervals in relation to STAFF.

The purpose of this paper is to analyse chorus events where reflected components, are simultaneously observed by the wave experiments STAFF and WBD, and to establish the characteristics of these reflected waves. In order to process STAFF-SA data, a dedicated software named PRASSADCO (PRopagation Analysis of STAFF-SA Data with COherency tests) is used to determine propagation characteristics of the observed emissions (Santolík and Parrot, 1998, 1999; Santolík, 2001; Santolík et al., 2001; Parrot et al., 2003a, b; Santolík et al., 2003a). A ray tracing program is operated to emulate the trajectories of the waves in the magnetosphere (Cerisier, 1970; Cairo and Lefeuvre, 1986; Muto and Hayakawa, 1987; Parrot et al., 2003b). This ray tracing software uses a diffusive equilibrium model for electron density. Two selected events are presented and analysed in Sect. 2 . They consist of chorus emissions with reflected components the nature and the propagation characteristics of which will be determined. Discussions and conclusions are given in Sect. 3.

\section{Detailed analysis of events recorded by CLUSTER}

\subsection{Chorus event recorded on 19 November 2001}

Figure 1 presents data recorded by STAFF-SA on 19 November 2001. The first four panels concern the power spectral densities of the magnetic components (arithmetic average of the three components) for $\mathrm{SC} 1, \mathrm{SC} 2, \mathrm{SC} 3$ and $\mathrm{SC} 4$, respectively. A banded electromagnetic emission of chorus type is seen between $100 \mathrm{~Hz}$ and $4 \mathrm{kHz}$, the maximum frequency being obtained when the satellite is near the magnetic equator, where equatorial noise is also observed around $100 \mathrm{~Hz}$ (Santolík et al., 2002), but these emissions are not related to our study and they will not be considered here. It is observed that SC3 is $40 \mathrm{~min}$ late relative to the three others. The degree of polarisation obtained from the eigenvalues of the magnetic spectral matrix has been calculated (not shown); a value close to 1.0 corresponds to the presence of a single plane wave. The value for the banded emission is $\sim 0.8$. This high value permits one to have confidence in the results of the wave polarisation and propagation parameters. An emission between 500 and $1000 \mathrm{~Hz}$ with an identical value of the degree of polarisation is observed in the time interval 12:00-13:00 UT on SC1, SC2 and SC4, and in the time interval 12:30-13:30 UT on SC3. It corresponds to less intense emissions in the power spectral density panels. The four middle panels of Fig. 1 give an estimation of the sign of the parallel component of the Poynting vector relative to the Earth's magnetic field $\mathrm{B}_{0}$ (Santolík et al., 2001; Parrot et al., 2003a). The spacecraft coordinates at the bottom of Fig. 1 are related to SC1. In the Poynting vector panels, a blue colour indicates that the wave vector is in the opposite direction of the Earth's magnetic field $\mathrm{B}_{0}$, i.e. directed towards the south, whereas the red colour is related to wave vectors in the direction of $\mathrm{B}_{0}$, i.e. directed towards the north. It is observed on all SC for the main intense chorus emissions that the colour changes close to the equator, which indicates that the chorus waves propagate away from the equator. In these four middle panels, it is also observed that the emissions with a lower intensity but with a degree of polarisation similar to the main emissions, propagate in an opposite direction. These emissions are directed toward the equator (the emission is also observed after the equator crossing), and it has been shown in a similar event that they are related to the main intense one; they are reflected waves (Parrot et al., 2003b). The last four panels of Fig. 1 present an estimation of the 2-D degree of polarization in the plane of maximum variance of the wave magnetic field (Santolík et al., 2003a). The 2-D degree of polarization tends to zero, with increasing presence of waves with different normal directions. It is observed that the degree of polarization of waves coming back toward the equator has similar values (between 0.6 and 1) as for the waves directly propagating from the source.

Figure 2 represents the spectrogram of the polar angle $\theta$ between the wave normal direction and the Earth's magnetic field $\mathrm{B}_{0}$ during the same time interval as Fig. 1 for all SC. These values are obtained with a Singular Value 


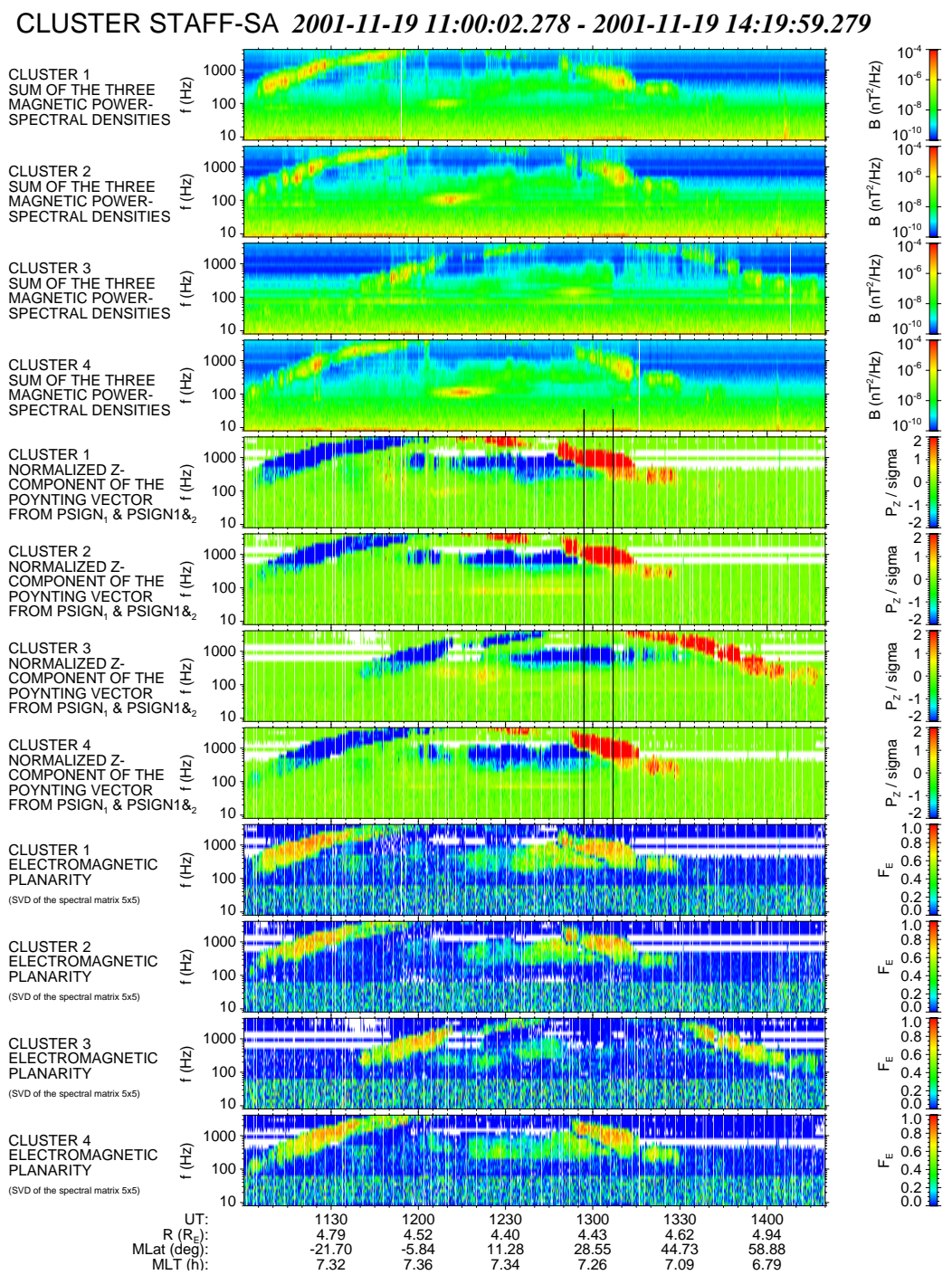

Fig. 1. Chorus event recorded on 19 November 2001 between 11:00 and 14:20 UT. From the top to the bottom, spectrogram of magnetic power spectral densities, z-component of the Poynting vector and degree of planar polarization are represented for the four SC, respectively. Values are color-coded according to the scales on the right. The two vertical lines indicate the time interval where the ray tracing is done.

CLUSTER STAFF-SA 2001-11-19 11:00:02.278 - 2001-11-19 14:19:58.159

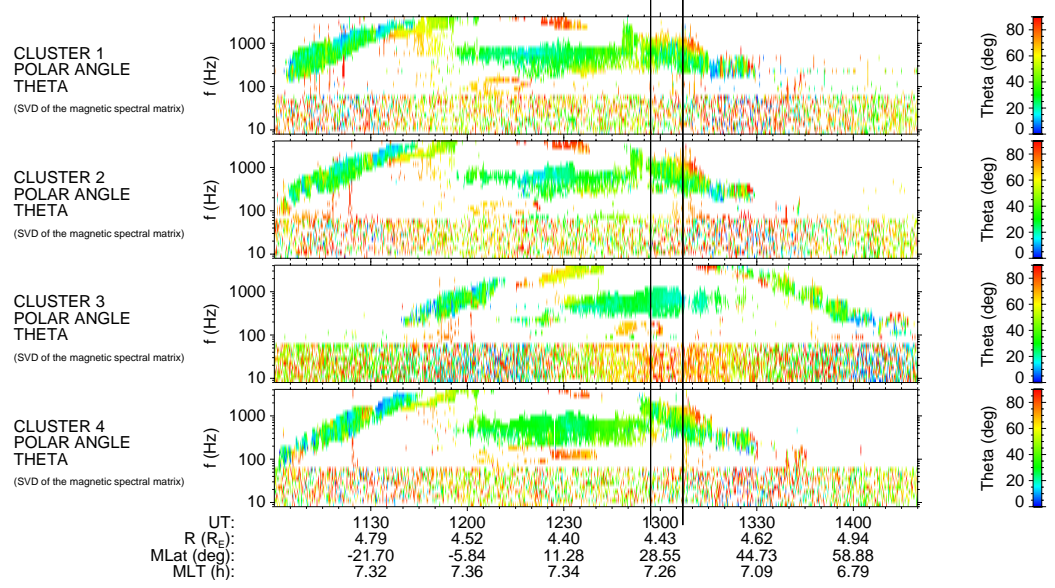

Fig. 2. Spectrograms of the wave normal angles for the event of Fig. 1 for the four SC. Values are color-coded according to the scales on the right. The two vertical lines indicate the time interval where the ray tracing is done. 


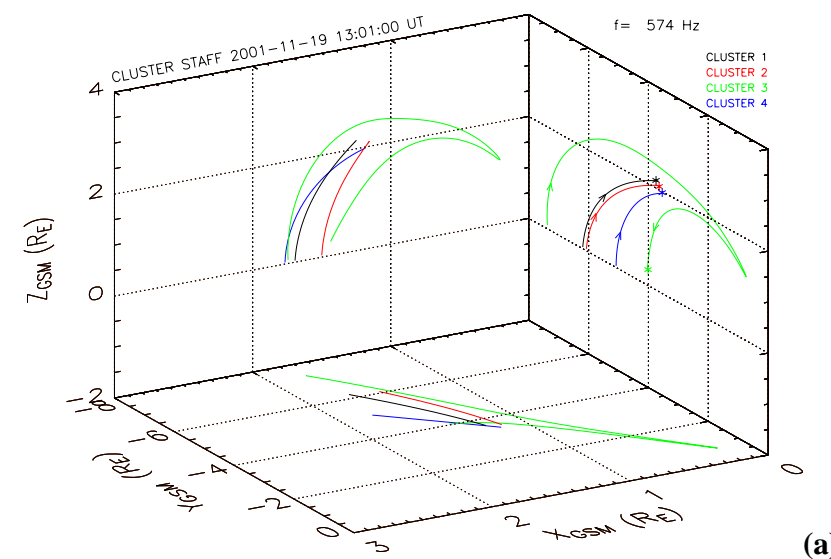

(a)

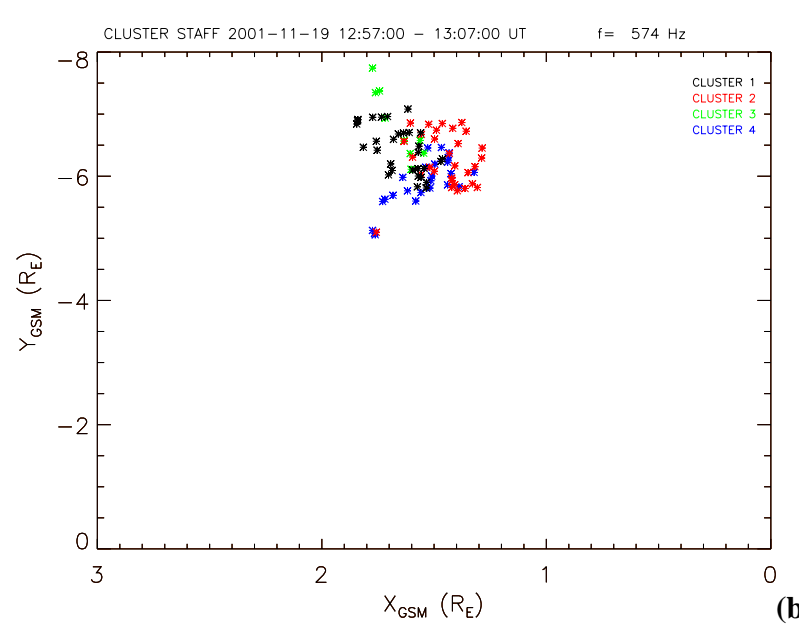

(b)

Fig. 3. Results of the ray tracing performed on 19 November 2001 at a frequency equal to $574 \mathrm{~Hz}$. (a) 2-D projections of one 3-D trace are represented for each SC according to their allocated colors at 13:01 UT. The arrows in the plane YZ indicate the real trajectory of the waves. The stars indicate the positions of the SC. (b) Location of the equator crossing in the equatorial plane for each SC between 12:57 and 13:07 UT.

Decomposition (SVD) method (Santolík et al., 2003a). They are color-coded from $0^{\circ}$ to $90^{\circ}$ according to the scale on the right. Due to the frequency limitation of STAFF-SA the $\theta$-values of the main chorus emission close to the generation zone (the magnetic equator) cannot be determined, but it can be seen that the waves below $1 \mathrm{kHz}$ which return to the equator (blue area in the Northern Hemisphere in the middle four panels of Fig. 1) cross this magnetic equator with low $\theta$-values $\left(<20^{\circ}\right)$. The data of Fig. 2 will be used with the corresponding azimuthal angles (not shown) as input for the ray tracing.

The results of multiple ray tracing for each satellite and for each polar and azimuthal determination of the wave normal direction between 12:57 and 13:07 UT are given in Fig. 3. During this time interval, which is indicated in Figs. 1 and 2, SC1, SC2 and SC4 observe chorus waves which escape from the equator, and $\mathrm{SC} 3$ observes at the same frequency an emission which returns to the equator. Figure $3 \mathrm{a}$ represents the projection of the 3-D traces of the rays on the 3

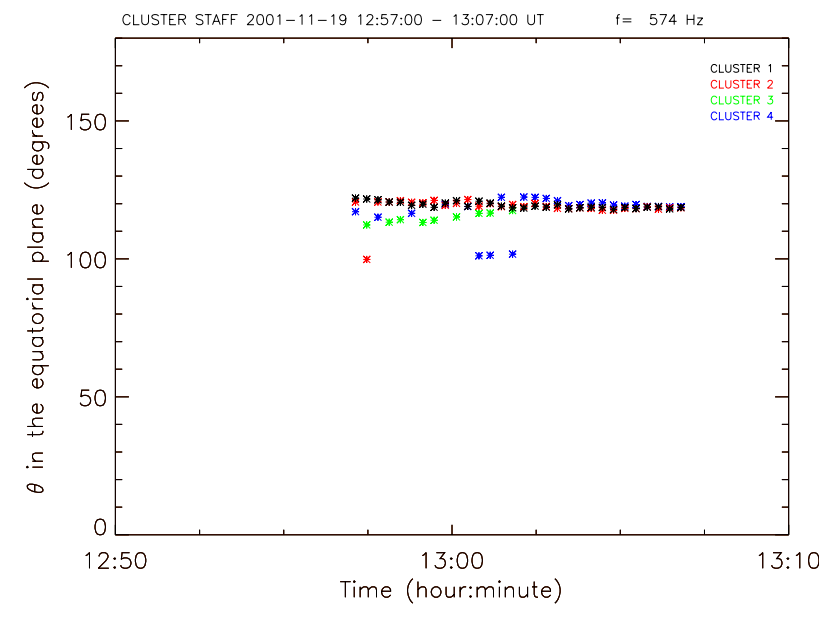

Fig. 4. Representation of the polar angle $\theta$ in the equatorial plane given by the ray tracing software as a function of time (it is the same time interval as Fig. 3) for each SC at $574 \mathrm{~Hz}$.

planes XY, XZ and YZ, in GSM coordinates for $574 \mathrm{~Hz}$. In order to have a better view of their trajectory, a single set of 4 rays is used in Fig. 3a. The four rays correspond to each SC and are colour coded according to the dedicated SC colours seen on the right upper corner. They are calculated at the same given time: 13:01 UT. For SC1, SC2 and SC4, the origin of the rays (position of the satellites) is nearly the same $\left(\mathrm{X}_{G S M} \sim 1.25 \mathrm{R}_{E}, \mathrm{Y}_{G S M} \sim-3.5 \mathrm{R}_{E}, \mathrm{Z}_{G S M} \sim 2.1 \mathrm{R}_{E}\right)$. The backward ray tracing software is stopped when the rays encounter the equator. SC3 is late relative to the three others, and it has just crossed the equator before 12:57 UT (Mlat $=2.97^{\circ}$ at 13:00 UT). The origin of its ray is $\mathrm{X}_{G S M} \sim 1.45 \mathrm{R}_{E}, \mathrm{Y}_{G S M} \sim-4.0 \mathrm{R}_{E}, \mathrm{Z}_{G S M} \sim 0.2 \mathrm{R}_{E}$ and it is observed that the ray only reaches the equator after a reflection at low altitudes. This is underlined in the plane $\mathrm{YZ}$ where the positions of the SC are indicated by stars and where the real trajectories of the waves which come from the equator are pointed out by arrows. Figure $3 b$ represents a cross section of all the ray trajectories in the equatorial plane for all SC between 12:57 and 13:07 UT. It is clearly shown on these plots that the waves observed, on one hand, by $\mathrm{SC} 3$, and on the other hand, by SC1, SC2 and SC4 span the same region in the equatorial plane (the source region of chorus). Waves that originate from the equator are first seen by $\mathrm{SC} 1, \mathrm{SC} 2$ and SC4. After that, they are going to higher latitudes where they suffer a LHR (Lower Hybrid Resonance) reflection at low altitudes. The reflection occurs when the frequency of the wave approaches the local LHR frequency (Kimura, 1966). At the end, they return to the equator at a different L-value. On their way back, they are observed by SC3.

This ray tracing software also provides information on the propagation and particularly on the wave normal along the ray path. Figure 4 displays the polar angle of the wave normal with the magnetic field as a function of the time when the rays cross the magnetic equator. The plot is done at a 

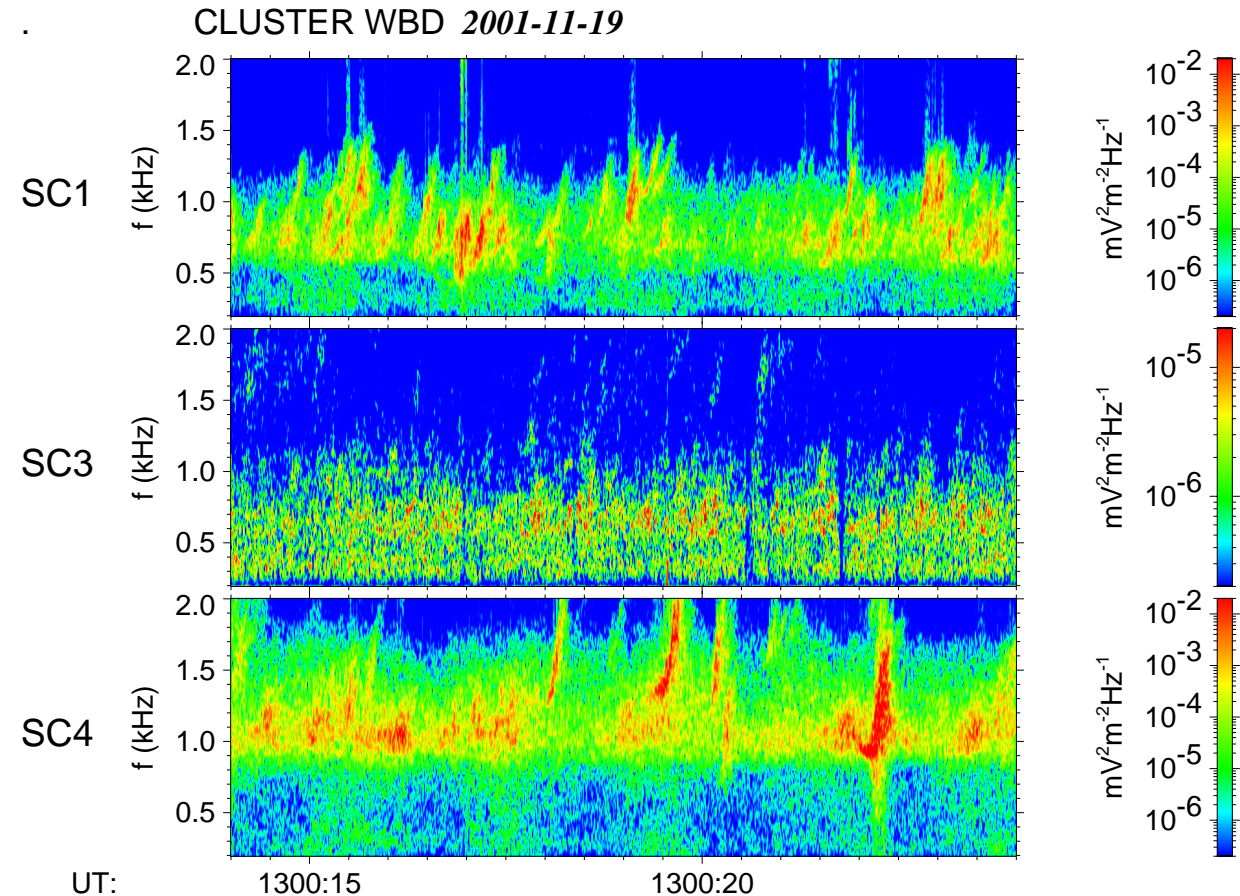

Fig. 5. Spectrograms of one electric component recorded by WBD on 19 November 2001 between 13:00:14 and 13:00:24 UT for SC1 (top), SC3 (middle) and SC4 (bottom).

frequency of $574 \mathrm{~Hz}$, and it is shown that the polar angle is rather equal to a constant value $\left(\sim 120^{\circ}\right)$ during the entire time interval and for all SCs including SC3.

Figure 5 represents the spectra of the electric data recorded by WBD between 13:00:14 and 13:00:24, i.e. during a selected period of $10 \mathrm{~s}$ at the time of the previously described ray tracing. On this day, three SCs are available: SC1 in the top panel, SC3 in the middle panel and SC4, in the bottom panel. The frequency range has been limited to the interesting part of the spectra $(0.2-2.0 \mathrm{kHz})$. The plots in the top (SC1) and bottom (SC4) clearly show chorus emissions which correspond to waves escaping from the equator if we consider the direction of the Poynting vector given in the middle panels of Fig. 1. It can be noticed that these two emissions are not exactly at the same frequency. Similar effects have been reported by Gurnett et al. (2001). In our case the explanation could be based on different positions of the two spacecraft. Unlike in the case of Gurnett et al. (2001), there is no correspondence of separate chorus elements observed by SC1 and SC4 and the sources probably are at different L-shells. Nevertheless, the plot in the middle panel (SC3) of Fig. 5 shows an emission which does not seem to contain separate chorus elements but still demonstrates some internal structure in the time-frequency plane. This emission corresponds to waves which return towards the equator, as it has been shown in Figs. 1 and 3. It must be noticed that the intensity of this emission is much lower (three orders) than the escaping chorus emissions shown on SC1 and SC4. This is in agreement with the observation reported by Parrot et al. (2003b) for a similar event.

\subsection{Chorus event recorded on 7 November 2001}

The second chorus event was recorded on 7 November 2001 between 13:00 and 17:00 UT. Figure 6 is the equivalent of Fig. 1 for this event. The first four panels show a banded chorus emission between $100 \mathrm{~Hz}$ and $4 \mathrm{kHz}$. The maximum intensity is obtained in the low frequency part of the emission up to $1 \mathrm{kHz}$. SC3 is $50 \mathrm{~min}$ late relative to the three others. The four middle panels of Fig. 6 indicate that these chorus waves are escaping from the equator. Emissions propagating in opposite direction are also observed between $500 \mathrm{~Hz}$ and $1 \mathrm{kHz}$ on all SC mainly in the Northern Hemisphere. The last four panels of Fig. 6 indicate that these former waves have a 2-D degree of polarization sufficiently high to be considered as single waves. Figure 7 is equivalent to Fig. 2 for the 7 November 2001 event. Again, the $\theta$-values of the main chorus emission close to the generation zone cannot be observed, but it is shown that the waves which return to the equator cross this equator with low $\theta$-values $\left(<20^{\circ}\right)$, as it is in Fig. 2.

As for the first event, a backward ray tracing has been performed between 15:25 and 15:49 UT (see Figs. 6 and 7), a time interval where SC1, SC2 and SC4 observe chorus waves which escape from the equator, and SC3 observes at the same frequency an emission which returns to the equator. The results are represented in Fig. 8. In the top, the projections of all the rays have been represented during the given time interval. These rays show that the emission observed on SC3 which returns to the equator has the same origin as the direct emissions observed by SC1, SC2 and SC4. During this 


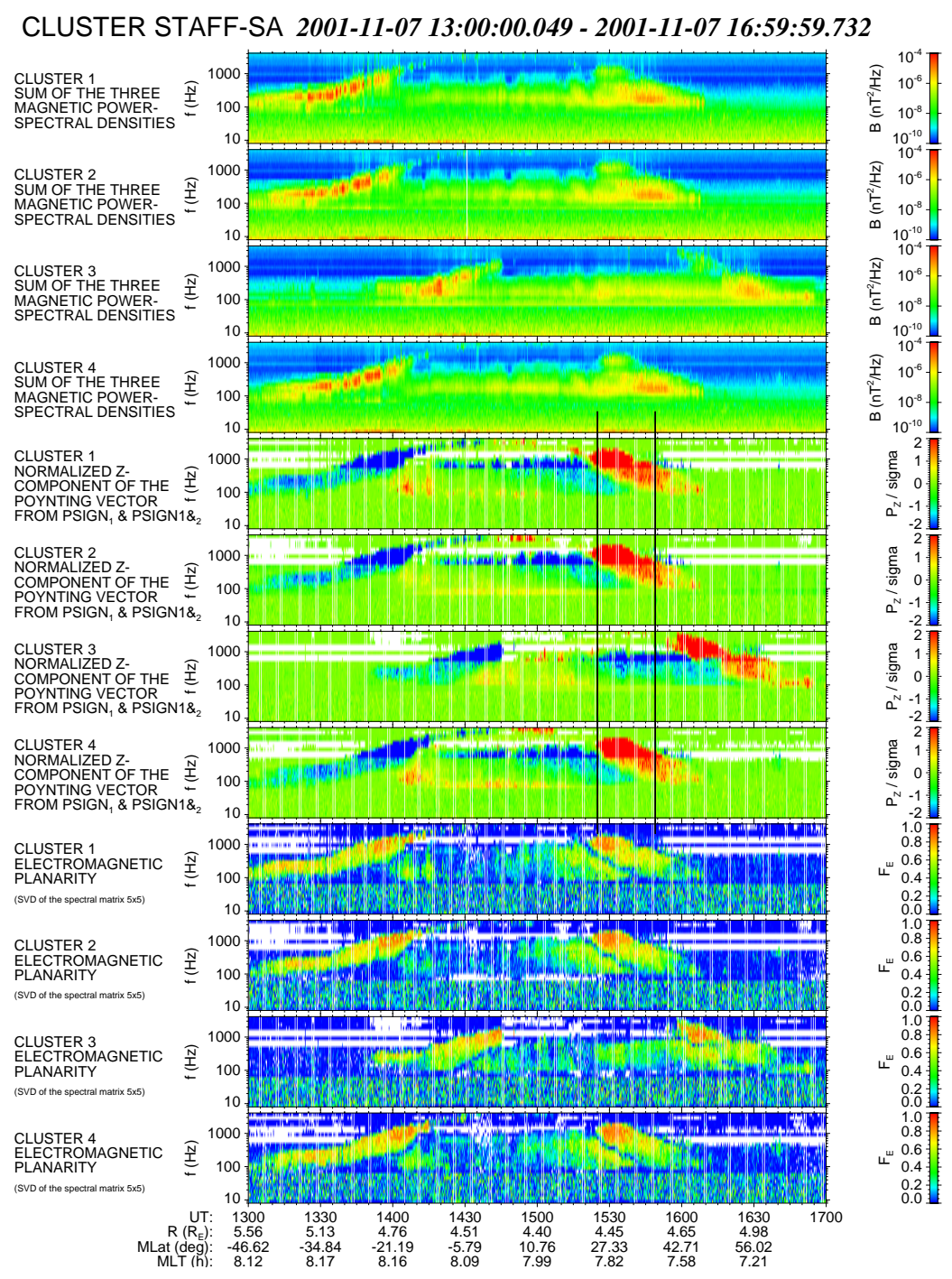

Fig. 6. Same as Fig. 1 but for the chorus event recorded on 7 November 2001 between 13:00 and 17:00 UT.

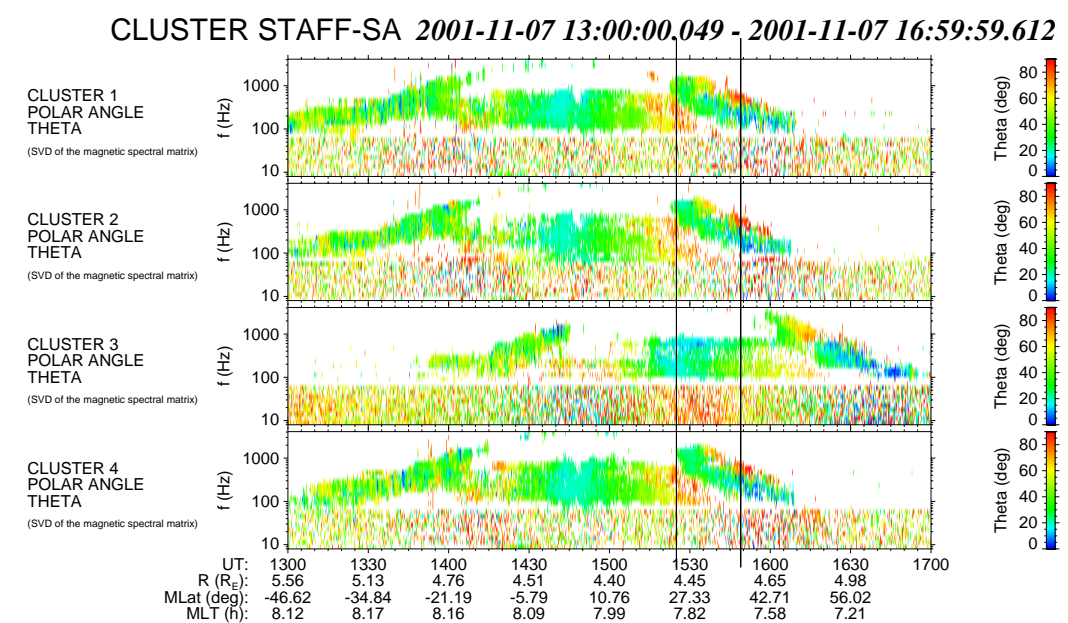

Fig. 7. Same as Fig. 2 but for 7 November 2001. 
time interval SC3 is very close to the equatorial plane. The ray locations of the equator crossing in the equatorial plane are plotted in the bottom of Fig. 8 for a frequency equal to $574 \mathrm{~Hz}$. It confirms the common origin of both emissions. The points located around $\left(\mathrm{X}_{G S M}=2.2 \mathrm{R}_{E}, \mathrm{Y}_{G S M}=-4 \mathrm{R}_{E}\right)$ are those corresponding to waves directly observed by SC3. Figure 9 displays the polar angle as a function of the time when the rays cross the magnetic equator at a frequency of $574 \mathrm{~Hz}$. The values of interest for SC1, SC2 and SC4 are around $120^{\circ}$ as in the previous event. The values for SC3 are a little bit lower $\sim 115^{\circ}$ (the higher green values $\left(>150^{\circ}\right.$ ) correspond to reflected waves observed directly by SC3).

During this event, WBD recorded data only on SC1. Then it is not possible to check the nature of the waves which return to the equator during our time interval of analysis. We have selected another interval on SC1 corresponding to reflected waves between 14:47:22 and 14:47:28 UT. The WBD spectrogram in the frequency range $0-1 \mathrm{kHz}$ of one electric component is shown on Fig. 10. Around $200 \mathrm{~Hz}$, we observe a rather continuous emission that looks like hiss with some discrete elements emerging from the hiss band. More interesting is the emission around $900 \mathrm{~Hz}$ because it exactly corresponds to the reflected waves. Discrete elements that have the appearance of chorus are observed. Figure 11 represents the power spectral densities observed by STAFF-SA during the same time interval as Fig. 10 and as a function of the frequency. The top panel concerns average values from the three magnetic components and the bottom panel is related to average values from the two electric components. The dashed line in the bottom panel represents the time averaged spectrum of the WBD data shown in Fig. 10. This figure confirms that STAFF-SA sees the same emissions detecting strong waves around $200 \mathrm{~Hz}$ and much weaker waves around $900 \mathrm{~Hz}$ (the peaks around $70 \mathrm{~Hz}$ are internal interferences).

\section{Summary and discussion}

We have shown a detailed multipoint study of two cases of whistler mode waves propagating toward the equator. In both cases, we have simultaneously observed intense chorus emissions propagating away from the equator. After the first analysis of these waves by Parrot et al. (2003b), we have presented here two other possible candidates of magnetospherically reflected chorus. Our interpretation is supported by a combination of different experimental and theoretical results based on the data from the four-spacecraft Cluster project.

We have used measured wave vector directions to initiate the backward ray calculations from the four different spacecraft. We have selected time intervals where three of the spacecraft recorded intense chorus emissions and one spacecraft observed weaker waves at the same frequency coming back to the equator. The simulated rays originating from this last spacecraft clearly show magnetospheric reflection after which they return back to lower latitudes and eventually cross the equatorial plane. The traces from three other spacecraft directly return back to the equator. We further show that both

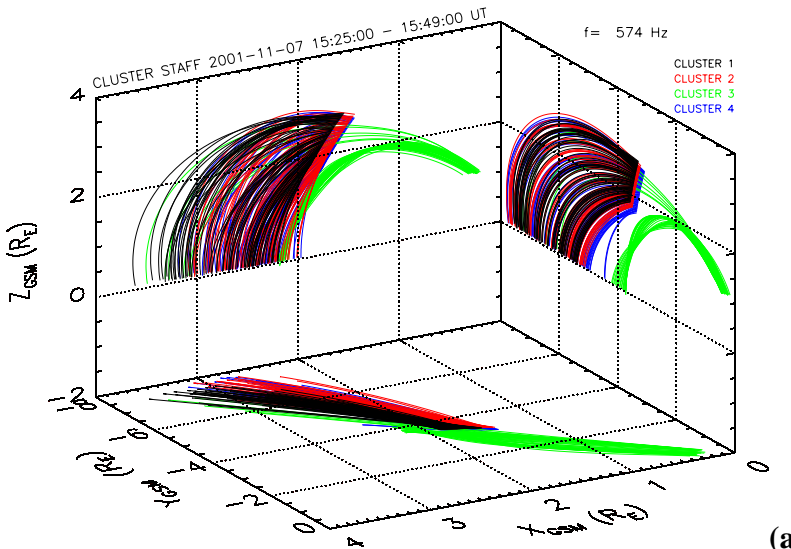

(a)

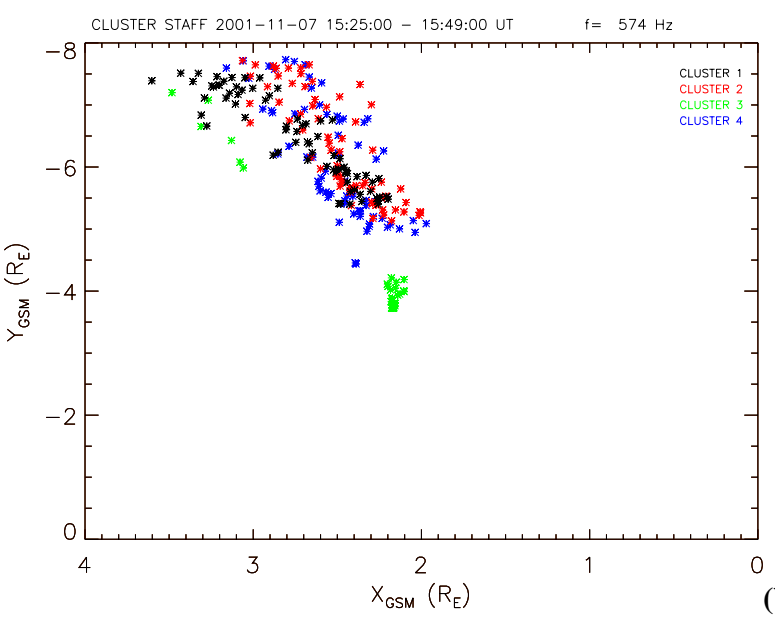

Fig. 8. Results of the ray tracing performed on 7 November 2001 between 15:25 and 15:49 UT at a frequency equal to $574 \mathrm{~Hz}$. (a) In comparison to Fig. 3a, here all the rays are represented during the given time interval. (b) Location of the equator crossing in the equatorial plane for each SC.

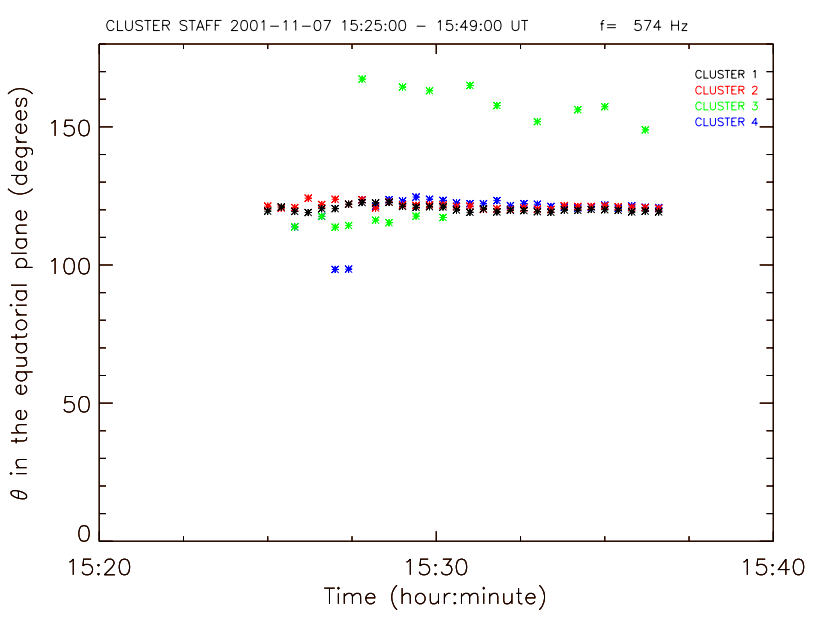

Fig. 9. Same as Fig. 4 but for 7 November 2001 between 15:25 and 15:49 UT. 


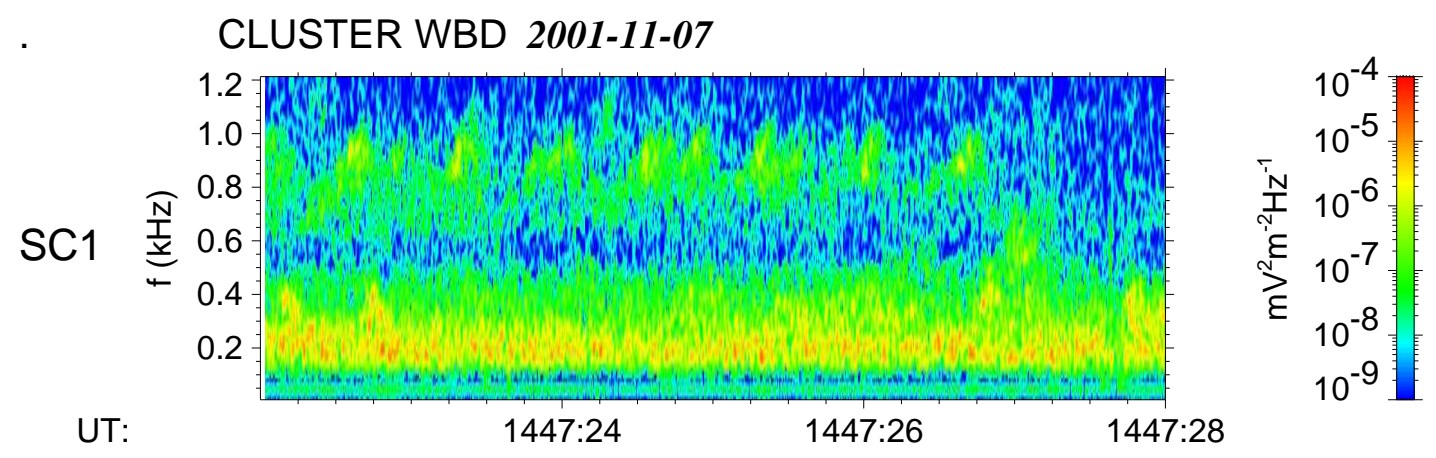

Fig. 10. Spectrogram of one electric component recorded by WBD on 7 November 2001 between 14:47:22 and 14:47:28 UT for SC1.

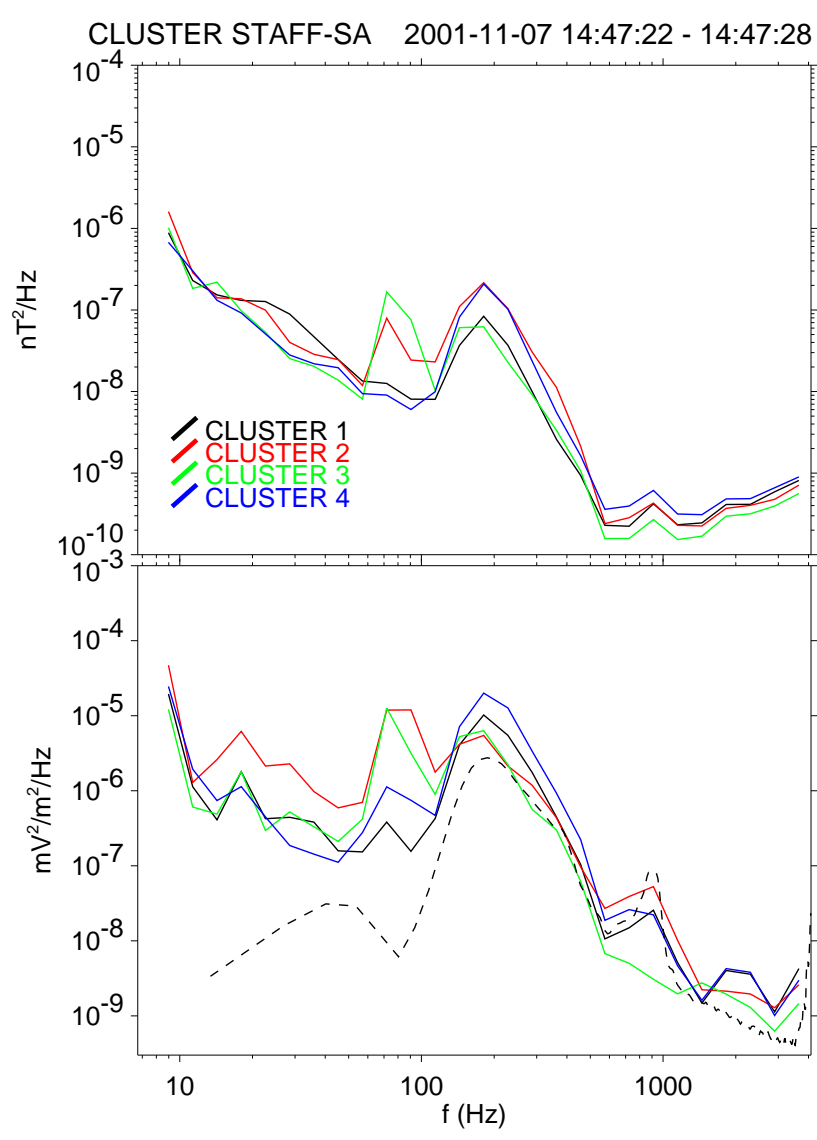

Fig. 11. Magnetic (top) and electric (bottom) power spectral densities recorded by STAFF-SA for each SC as a function of frequency during the same time interval as Fig. 10. The dashed line in the bottom panel is from WBD.

the reflected and direct rays cross the equatorial plane in the same region, and that, in this plane, their computed wavenormal angles are inclined by approximately the same angle of $60^{\circ}$ from the local magnetic field line.

This is an important result supporting the idea of magnetospherically reflected chorus. Both direct and reflected waves can originate from the same source, both escaping the source region at similar oblique wave-normal angles. This result, however, needs some discussion since the chorus is usually supposed to propagate parallel to the field lines close to its source (e.g. Sazhin and Hayakawa, 1992) in its lowerfrequency band. One possible explanation could be that the waves really are unstable at these oblique angles. This possibility still has little theoretical background, in spite of some experimental indications concerning the plasmaspheric hiss (Storey et al., 1991). Another explanation could be based on recent results on the transverse size of chorus sources which appeared to be of the order of the wavelength in the case analyzed by Santolík and Gurnett (2003). This may imply a sufficiently large interval of initial wave-normal angles of the waves escaping from the source, containing parallel and oblique directions, as has also been found by Chum et al. (2004) in their recent ray tracing study.

Other important new results of this paper are indications of a "smoothed" fine time-frequency structure of the reflected waves visible on the detailed power spectrograms. The separate chorus-like elements can sometimes be roughly visible but these features are most of the time hidden in a hisslike background. In distinction from the plasmaspheric hiss which appears shapeless on the spectrograms, these reflected waves still manifest an internal bursty structure. If we accept the hypothesis that these waves are in fact reflected chorus, the observations can be explained by degeneration of the chorus time-frequency structure during its propagation. The dispersion effects may cause similar modifications, as have been studied for magnetospherically reflected whistlers by Shklyar and Jiříček (2000), and gradually move the appearance of time-frequency spectrograms to a more hiss-like shape. This very same idea has been initially proposed by H. C. Koons (see page 19486 of Storey et al., 1991) as an alternative explanation of the origin of plasmaspheric hiss, and further developed by Draganov et al. (1992). Under this hypothesis, plasmaspheric hiss is explained by the accumulation of whistlers propagating in the plasmasphere. The relation of hiss and chorus is, however, more complex and hiss bands also have been considered as sources of the chorus emissions (Koons, 1981).

Finally, we have demonstrated with the experimental data that the reflected waves return to the equatorial plane with wave vectors oriented nearly parallel to the field lines. These 
wave vector directions may be favourable to further amplification by cyclotron resonance with radiation belt electrons (Kennel and Petschek, 1966). If these waves are able to penetrate the plasmapause toward lower altitudes, they could provide another possible embryonic source of the plasmaspheric hiss. Verification of this mechanism is, however, beyond the scope of the present paper, and will be the subject of future investigations.

Acknowledgements. Thanks are due to the PIs of the FGM (A. Balogh), WHISPER (P. Décreau), and EFW (M. André) experiments for the use of their data and to the Hungarian Cluster Data Center, which provides auxiliary data. The STAFF-SA data were available thanks to C. Harvey and M. Maksimovic. We acknowledge discussions with A. G. Demekhov on the possible radiation pattern of chorus sources. ESA and CNES are thanked for their support for the STAFF experiment. Portions of this work were performed at the University of Iowa under the NASA Goddard Space Flight Center Grant No. NAG5-9974. O. Santolík acknowledges additional support from grants MSM 113200004 and GACR 202/03/0832.

Topical Editor T. Pulkkinen thanks N. Meredith and another referee for their help in evaluating this paper.

\section{References}

Burtis, W. J. and Helliwell, R.A.: Banded chorus: A new type of VLF radiation observed in the magnetosphere by OGO-1 and OGO-3, J. Geophys. Res., 74, 3002-3010, 1969.

Burton, R. K. and Holzer, R. E.: The origin and propagation of chorus in the outer magnetosphere, J. Geophys. Res., 79, 10141023, 1974.

Cairo, L. and Lefeuvre, F.: Localization of sources of ELF/VLF hiss observed in the magnetosphere: Three-dimensional ray tracing, J. Geophys. Res., 91, 4352-4364, 1986.

Cerisier, J. C.: Propagation perpendiculaire au voisinage de la fréquence de la résonance hybride basse, in Plasma Waves in Space and in the Laboratory, Edinburgh University Press, Edinburgh, 2, 487-521, 1970.

Chum, J., Jiříček, F., Šmilauer, J., and Shklyar, D.: Magion 5 observation of chorus-like emissions and their propagation features as inferred from the ray tracing simulation, Ann. Geophys., in press, 2004.

Cornilleau-Wehrlin, N., Chauveau, P., Louis, S., Meyer, A., Nappa, J. M., Perraut, S., Rezeau, L., Robert, P., Roux, A., de Villedary, C., de Conchy, Y., Friel, L., Harvey, C. C., Hubert, D., Lacombe, C., Manning, R., Wouters, F., Lefeuvre, F., Parrot, M., Pinçon, J. L., Poirier, B., Kofman, W., Louarn, P., and the STAFF investigator team: The CLUSTER STAFF experiment (Spatio Temporal Analysis of Field Fluctuations Experiment), Space Science Rev., 79, 107-136, 1997.

Cornilleau-Wehrlin, N., Chanteur, G., Rezeau, L., Parrot, M., Pinçon, J. L., and STAFF team: The CLUSTER STAFF experiment specific multi-point analysis, Proc. CLUSTER-II Workshop on Multiscale/Multipoint Plasma Measurements, London, 22-24 September 1999, ESA SP-449, February 2000.

Cornilleau-Wehrlin, N., Chanteur, G., Perraut, S., Rezeau, L., Robert, P., Roux, A., de Villedary, C., Canu, P., Maksimovic, M., de Conchy, Y., Hubert, D., Lacombe, C., Lefeuvre, F., Parrot, M., Pinçon, J. L., Décréau, P. M. E., Harvey, C. C., Louarn, Ph., Kofman, W., Santolík, O., Alleyne, H. St. C., Roth, M., and
STAFF team: First results obtained by the Cluster STAFF experiment, Ann. Geophys., 21, 2, 437, 2003.

Dunkel, N. and Helliwell, R.: Whistler mode emissions in the OGO 1 satellite, J. Geophys. Res., 74, 6371-6385, 1969.

Draganov, A. B., Inan, U. S., Sonwalkar, V. S., and Bell, T. F.: Magnetospherically reflected whistlers as a source of plasmaspheric hiss, Geophys. Res. Lett., 19, 233-236, 1992.

Goldstein, B. E. and Tsurutani, B. T.: Wave normal directions of chorus near the equatorial source region, J. Geophys. Res., 89, 2789-2810, 1984.

Gurnett, D. A., Huff, R. L., Pickett, J. S., Persoon, A. M., Mutel, R. L., Christopher, I. W., Kletzing, C. A., Inan, U. S., Martin, W. L., Bougeret, J.-L., Alleyne, H. St. C., and Yearby, K. H.: First results from the CLUSTER wideband plasma wave investigation, Ann. Geophys., 19, 1259-1272, 2001.

Gustafsson, G., André, M., Carozzi, T., Eriksson, A. I., Fälthammar, C.-G., Grard, R., Holmgren, G., Holtet, J. A., Ivchenko, N., Karlsson, T., Khotyaintsev, Y., Klimov, S., Laakso, H. Lindqvist, P.-A., Lybekk, B., Marklund, G., Mozer, F., Mursula, K., Pedersen, A., Popielawska, B., Savin, S., Stasiewicz, K., Tanskanen, P., Vaivads, A., and Wahlund, J.-E.: First results of electric field and density observations by Cluster EFW based on initial months of operation, Ann. Geophys., 19, 1219-1240, 2001.

Helliwell, R. A.: A theory of discrete emissions from the magnetosphere, J. Geophys. Res., 72, 4773-4790, 1967.

Kennel, C. F. and Petscheck, H. E.: Limit on stably trapped particle fluxes, J. Geophys. Res., 71, 1-28, 1966.

Kimura, I.: Effects of ions on whistler-mode ray tracing, Radio Science, 1, 3, 269-283, 1966.

Koons, H. C.: The role of hiss in magnetospheric chorus emissions, J. Geophys. Res., 86, 8, 6745-6754, 1981.

Ledocq, M. J., Gurnett, D. A., and Hospodarsky, G. B.: Chorus source locations from VLF Poynting flux measurements with the Polar spacecraft, Geophys. Res. Lett., 25, 21, 4063, 1998.

Meredith, N. P., Horne, R. B., and Anderson, R. R.: Substorm dependence of chorus amplitudes: Implications for the acceleration of electrons to relativistic energies, J. Geophys. Res., 106, 13 165-13 178, 2001.

Muto, H. and Hayakawa, M.: Ray-tracing study of the propagation in the magnetosphere of whistler-mode VLF emissions with frequency above one half the gyrofrequency, Planet. Space Sci., 35, 1397-1404, 1987.

Nagano, I., Yagitani, S., Kojima, H., and Matsumoto, H.: Analysis of wave normal and Poynting vectors of the chorus emissions observed by Geotail, J. Geomagn. Geoelectr., 48, 299-307, 1996.

Parrot, M., Santolík, O., Cornilleau-Wehrlin, N., Maksimovic, and M., Harvey, C. C.: Source location of chorus emissions observed by CLUSTER, Annales Geophys., Ann. Geophys., 21, 2, 473 480, 2003a.

Parrot, M., Santolík, O., Cornilleau-Wehrlin, N., Maksimovic, M., and Harvey, C. C.: Magnetospherically reflected chorus waves revealed by ray tracing with CLUSTER data, Ann. Geophys., 21, 5, 1111-1120, 2003b.

Santolík, O.: Propagation Analysis of Staff-SA Data with Coherency Tests, LPCE/NTS/073.C, Lab. Phys. Chimie Environ./CNRS, Orléans, France, 2001.

Santolík, O. and Parrot, M.: Propagation analysis of electromagnetic waves between the helium and proton gyro-frequencies in the low-altitude auroral zone, J. Geophys. Res., 103, 20469 $20480,1998$. 
Santolík, O. and Parrot, M.: Case studies on wave propagation and polarisation of ELF emissions observed by Freja around the local proton gyro-frequency, J. Geophys. Res., 104, 2459-2476, 1999.

Santolík, O., Lefeuvre, F., Parrot, M., and Rauch, J. L.: Complete wave-vector directions of electromagnetic emissions: Applications to INTERBALL-2 measurements in the night-side auroral zone, J. Geophys. Res., 106, 13 191-13 201, 2001.

Santolík, O., Pickett, J. S., Gurnett, D. A., Maksimovic, M., Cornilleau-Wehrlin, N.: Spatio temporal variability and propagation of equatorial noise observed by Cluster, J. Geophys. Res., 107, A12, 1495, doi:10.1029/2001JA009159, 2002.

Santolík, O. and Gurnett, D. A.: Transverse dimensions of chorus in the source region, Geophys. Res. Lett., 30, 1031, doi:10.1029/2002GL016178, 2003.

Santolík, O., Parrot, M., and Lefeuvre, F.: SVD methods for wave propagation analysis, Radio Science, 38, doi:10.1029/2000RS002523, 2003a.

Santolík, O., Gurnett, D. A., Pickett, J. S., Parrot, M., and Cornilleau-Wehrlin, N.: Spatio-temporal structure of storm-time chorus, J. Geophys. Res., 108(A7), 1278, doi:10.1029/2002JA009791, 2003b.

Sazhin, S. S. and Hayakawa, M.: Magnetospheric chorus emissions: A review, Planet. Space Sci., 40, 681-697, 1992.
Shklyar, D. R. and Jiříček, F.: Simulation of nonducted whistler spectrograms observed aboard the MAGION 4 and 5 satellites, J. Atm. Sol. Terr. Phys., 62, 347-370, 2000.

Storey, L. R. O., Lefeuvre, F., Parrot, M., Cairo, L., and Anderson, R. R.: Initial survey of the wave distribution functions for plasmaspheric hiss observed by ISEE 1, J. Geophys. Res., 96, 19469-19489, 1991.

Titova, E. E., Kozelov, B.V., Jiř́̌ček, F., Smilauer, J., Demekhov, A. G., and Trakhtengerts, V. Yu.: Verification of the backward wave oscillator model of VLF chorus generation using data from MAGION 5 satellite, Ann. Geophys., 21, 5, 1073, 2003.

Tsurutani, B. T. and Smith, E. J.: Postmidnight chorus: a substorm phenomenon, J. Geophys. Res., 79, 118-127, 1974.

Tsurutani, B. T. and Smith, E. J.: Two types of magnetospheric ELF chorus and their substorm dependences, J. Geophys. Res., 82, 5112-5128, 1977.

Tsurutani, B. T., Smith, E. J., West Jr., H. I., and Buck, R. M.: Chorus, Energetic electrons and magnetospheric substorms, in wave instabilities in space plasmas, edited by Palmadesso, P. J. and Papadopoulos, K., D., Reidel Publishing Company, 55-62, 1979. 